\title{
"Measuring Frailty Can Improve Shared-Decision Making between Patients and Physicians": An Experience of Measuring Frailty in Elderly Chronic Kidney Disease Patients
}

Mahira Ilyas. ${ }^{1}$

Frailty is commonly underdiagnosed and undertreated in Chronic Kidney Disease patients on dialysis partly because of the lack of a standardized definition. Current guidelines serve elderly patients poorly meaning we need a wider focus on new priorities of care when managing patients identified as frail. The focus on dialysis patients nearing the end of life should be on promoting a better quality, "adding life to years," instead of quantity of years.

\section{Introduction}

The prevalence of frailty in the elderly population is thought to be around $11 \%$, whereas the prevalence of frailty in elderly dialysis dependent Chronic Kidney Disease (CKD) patients is reported to be more than $60 \%$.

Frail renal patients face challenging treatment choices underpinned by ethical tensions when considering dialysis. Elderly CKD patients often have to make ongoing adjustments throughout their journey with the disease which involved accepting the life-threatening diagnosis, the need for life-long treatment, integrating the treatment into their lives as well as coping with the side effects and complications of dialysis. ${ }^{3}$ Therefore, identifying a frail patient in advance and optimizing the shared-decision making process can help these patients make wellinformed decisions based on a complete understanding of their condition whilst their personal values and preferences are also taken into account. ${ }^{2}$ Dialysis has become so common in the routine practice of medical care and in the biomedicalization of ageing, that it has made it challenging for patients, families and even healthcare professionals to make the right decisions. ${ }^{4}$

\section{Why is there a need to formally assess frailty?}

All chronic diseases are often difficult to accept. Elderly CKD patients have to make ongoing psychological adjustments throughout their journey with the disease, coping with the burden of treatments with their complications and accepting the lifestyle changes (such as diet) that they are required to make for treatment to be effective. This could be perceived as a part of life that we are reluctant to accept thus questions the need to formally assess frailty in order to identify those who may benefit from supportive care treatment, which improves the Quality of Life (QoL) instead of having dialysis, which improves the quantity of life.

\section{The Experience}

I have had the opportunity to work with nephrologists and geriatricians to assess frailty in patients over 60 years in the renal Low Clearance Clinic (LCC) at King's College Hospital. We have been assessing patients using various tools to measure falls risk, memory, mobility and checking if patients have any extra care needs. The aim of the assessment is to help the healthcare professionals, in both the renal and geriatric departments, to arrange follow up appointments where a comprehensive geriatric assessment can be undertaken to direct patients to helpful services such as a memory clinic.

The tools I have been using include: Rockwood Clinical Frailty Score, a frailty screening tool that relies solely on clinical judgement; Montreal Cognitive Assessment (MoCA), a brief 30 question test that evaluates various cognitive abilities; Prisma 7, a seven-item questionnaire to identify disability; and Timed-Up-And-Go (TUG), a mobility test measuring the time taken to walk 3 meters and back. Patients who had a MoCA score <24/30, TUG >15 seconds, or Rockwood score .5 were considered as frail and thus referred for a comprehensive geriatric assessment.

I have also had the privilege to sit in consultations between a consultant nephrologist and CKD patients, identified as frail, to see how shared decision-making is approached by doctors. Patients often believe that dialysis will "cure" their CKD or reverse their condition which unfortunately is not the case. As a result, patients feel positive about starting dialysis in the hope that it will alleviate all their symptoms, forgetting that most of their symptoms are caused by their comorbidities such as Diabetes, Ischemic Heart Disease, Depression, Dementia etc. and that dialysis does not remedy these associated conditions. In the consultations, I have observed doctors educate patients about the advantages and disadvantages of dialysis. I have felt that there is an apparent 'generational gap' between the frail and elderly patients compared to the younger patients on dialysis. The elderly patients often seem to be confused about the manifestations of their illness and that could be because of their traditional views that they completely agree with what they have heard from the doctor or are influenced by their families that dialysis is the right treatment for them. However, it should be made clear that the medical culture has evolved from disease-focused to patient-focused treatments. Nephrologists now carefully explain to patients why dialysis may not be appropriate for everyone, both clinically and from a QoL perspective. After this, the doctor respectfully listens to the patient's views and helps them decide if utilizing the supportive care pathway could improve their QoL more than dialysis could.

The supportive care pathway offers psychological, social and spiritual care for the patient and their family and conservatively manages the renal problems by delaying renal progression (e.g. controlling blood pressure, diabetes, cholesterol, avoiding nephrotoxins), minimizes cardiovascular risk and the active management of renal and non-renal symptoms. The aim of supportive care is to focus on the QoL and allow the patient's wishes to be heard. For example, if a frail End Stage Renal

\section{King's College London, London, United Kingdom.}




\section{Experience}

Disease patient wishes to attend their daughter's wedding abroad, the pathway empowers and supports the patient's wishes.

\section{Conclusion}

This experience that I have embarked on has been a great privilege in understanding the importance of measuring frailty in the older population with CKD. Evidence has shown that measuring frailty helps identify patients who will thrive under conservative management with a maintained QoL instead of experiencing a sharp decline in their life satisfaction as seen with dialysis. If frailty is identified, it will enable healthcare professionals to recognize and treat the complexity of symptom burden in the patient's active, supportive and palliative stages of CKD through supportive care. 


\section{References}

1. Johansen KL, Chertow GM, Jin C, Kutner NG. Significance of Frailty among Dialysis Patients. J Am Soc Nephrol. 2007 Nov; 18(11):2960-7.

2. Swidler M. Considerations in Starting a Patient with Advanced Frailty on Dialysis: Complex Biology Meets Challenging Ethics. Clin J Am Soc Nephrol. 2013 Aug;8(8):1421-8.
3. Coh ZS, Griva K. Anxiety and depression in patients with end-stage renal disease: impact and management challenges; a narrative review. Int J Nephrol Renovasc Dis. 2018 Mar 12;11:93-102.

4. Kaufman SR, Shim JK, Russ AJ. Revisiting the Biomedicalization of Aging: Clinical Trends and Ethical Challenges. Cerontologist. 2004 Dec;44(6):731-8.

\section{Acknowledgments}

I would like to acknowledge Dr Katie Vinen and Dr Ambika Nedungadi for welcoming me into their team and supporting me to undertake this experience. Conflict of Interest Statement it Funding

The Authors have no funding, financial relationships or conflicts of interest to disclose.

\section{Author Contributions}

Conceptualization: MI. Investigation: MI. Writing - Original Draft: MI. Writing - Review đt Editing: MI.

Cite as:

Ilyas M. "Measuring Frailty Can Improve Shared-Decision Making between Patients and Physicians": An Experience of Measuring Frailty in Elderly Chronic Kidney Disease Patients. Int J Med Students. 2019;7(1):18-20. 